\title{
Effect and safety of dual anti-human epidermal growth factor receptor 2 therapy compared to monotherapy in patients with human epidermal growth factor receptor 2-positive breast cancer: a systematic review
}

Xiao Zhang ${ }^{1+}$, Xin-Ji Zhang ${ }^{1+}$, Tian-Yi Zhang ${ }^{1+}$, Fei-Fei Yu' ${ }^{1}$, Xin Wei ${ }^{2}$, Ye-Sheng $\mathrm{Li}^{3}$ and Jia He ${ }^{1 *}$

\begin{abstract}
Background: Dual anti-human epidermal growth factor receptor 2 (HER2) therapies have been shown to improve outcomes of HER2-positive breast cancer patients. We undertook a systematic review to compare treatment outcomes for patients who received single or combined anti-HER2 therapies.

Methods: We identified randomized control trials that compared dual anti-HER2 therapy and anti-HER2 monotherapy in patients with HER2-positive breast cancer. Outcomes included pathologic complete response (pCR), overall survival (OS), progression-free survival (PFS), and adverse events. Included in the analysis were seven trials that recruited 2,609 patients.

Results: In the neoadjuvant setting, the pooled PCR rate in the dual anti-HER2 therapy and monotherapy groups in combination with chemotherapy was $54.8 \%$ and $36 \%$, respectively. This difference was statistically significant (relative risk, 1.56; $95 \%$ confidence interval $(\mathrm{Cl}), 1.23-1.97 ; \mathrm{p}<0.001)$. In the metastatic setting, dual anti-HER2 therapy demonstrated significant benefits in both PFS (hazard ratio (HR), $0.71 ; 95 \% \mathrm{Cl}, 0.62-0.81 ; \mathrm{p}<0.001$ ) and OS (HR, 0.68; $95 \% \mathrm{Cl}, 0.57-0.82 ; \mathrm{p}<0.001$ ). Subgroup analyses indicated that the addition of chemotherapy to dual anti-HER2 therapy could greatly improve PCR in the neoadjuvant settings. However, in the metastatic setting, similar PFS and OS were found in patients receiving dual anti-HER2 therapy with or without chemotherapy. Dual anti-HER2 therapy was associated with more frequent adverse events than monotherapy, but no statistical differences were observed in cardiac toxicity.
\end{abstract}

Conclusions: This systematic review provides a summary of all the data currently available, and confirms the benefits and risks of dual anti-HER2 therapy for HER2-positive breast cancer.

Keywords: anti-HER2 therapy, HER2-positive breast cancer, Systematic review

\footnotetext{
* Correspondence: hejia63@yeah.net

${ }^{\dagger}$ Equal contributors

'Department of Health Statistics, Second Military Medical University,

Shanghai, China

Full list of author information is available at the end of the article
} 


\section{Background}

Despite advances in early diagnosis and treatment, breast cancer remains a significant public health concern; more than million new cases are diagnosed each year, resulting in 400,000 deaths worldwide [1-3]. Human epidermal growth factor receptor 2 (HER2) protein is overexpressed in 15-20\% of all breast cancers (HER2-positive breast cancer) and is associated with a poor outcome $[4,5]$.

The development of trastuzumab, a monoclonal antibody against HER2, has dramatically changed the prognosis for HER2-positive breast cancer patients [6]. Multiple randomized controlled trials (RCTs) have shown that trastuzumab therapy improves patient outcomes, and consequently trastuzumab has become the standard treatment for HER2-positive breast cancer patients in both the neoadjuvant and metastatic settings [7,8]. Following trastuzumab, lapatinib, an anti-HER2 tyrosine kinase inhibitor, was approved for use, in combination with capecitabine, for the treatment of HER2-positive metastatic breast cancer that has progressed with standard treatment $[9,10]$.

Despite these improvements, resistance to these drugs remains a challenge, and novel therapeutic approaches are required $[11,12]$. The effect of combining different antiHER2-targeted agents is one therapeutic strategy currently under investigation [13]. Laboratory studies have shown that dual anti-HER2 therapy can block the signaling from HER2 and its related HER family members more completely, leading to increased cell death and tumor shrinkage in HER2-positive models of breast cancer [14]. The addition of pertuzumab, an anti-HER2 monoclonal antibody, to trastuzumab and docetaxel therapy significantly increases progression-free survival (PFS) for patients with HER2-positive metastatic breast cancer (median PFS, 19.5 versus 12.4 months) [15]. These impressive results have provided a strong rationale for conducting randomized controlled studies evaluating trastuzumab in combination with lapatinib or pertuzumab for HER2-positive breast cancer in both the adjuvant and metastatic settings. In this study, we conduct a systematic review of these RCTs to summarize the benefits and risks of dual anti-HER2 therapy, as compared with monotherapy, for HER2-positive breast cancer patients.

\section{Methods}

\section{Data sources, search strategy, and selection criteria}

The systematic review was performed according to the Quality of Reporting of Meta-analyses (QUORUM) guidelines [16]. We systematic searched PubMed, EmBase, MEDLINE, and the Cochrane Central Registered Controlled Trials for studies conducted prior to May 2013, using the following keywords: trastuzumab, pertuzumab, lapatinib, and breast cancer. The search was limited to randomized clinical trials, but without language restrictions. In addition, the American Society of Clinical
Oncology (ASCO) Annual Meeting proceedings and the San Antonio Breast Cancer Symposium Meeting abstracts from 2004 to 2013 were individually searched for relevant randomized clinical trials. An independent search of relevant reviews and meta-analyses regarding trastuzumab, pertuzumab, or lapatinib was also conducted to ensure that no studies were missed. We reviewed each publication, and only the most recent or complete report of clinical trials was included when duplicate publications were identified. Efforts were also made to contact the study authors when relevant data were not clear.

The goal of this study was to evaluate the efficacy and safety of dual anti-HER2 therapy compared to monotherapy. Thus, only RCTs comparing dual anti-HER2 therapy (lapatinib, trastuzumab, or pertuzumab) with anti-HER2 monotherapy, with or without chemotherapy, in breast cancer were eligible. Phase I trials and singlearm phase II trials were excluded because of an absence of controls. Eligible studies had to meet the following inclusion criteria: studies had to 1 ) be prospective phase II and III trials with HER2 breast cancer patients, and 2) assign patients randomly to anti-HER2 single agent or combination treatment. The literature search and selection were undertaken independently by two investigators (Xiao Zhang and Fei-Fei Yu), and any disagreement was investigated by a third investigator (Xin-Ji Zhang) until a consensus was reached.

\section{Data extraction and quality assessment}

Data extraction and collection was performed independently by two reviewers (Xiao Zhang and Fei-Fei Yu) using a standardized protocol. Disagreements were adjudicated by a third reviewer (Xin-Ji Zhang) after referring to the original articles. The following information was extracted from each included study for baseline characteristics: the first author's surname, publication year, original country, age, number of patients per arm, dose and duration of anti-HER2 therapy, type/dose of chemotherapy, median follow-up period, and outcome measures. End points of interest included overall survival (OS), PFS, pathologic complete response (pCR), and adverse events (AEs). The quality of the trials included in this study were assessed using the Jadad scale [17]. The trials were assessed on the basis of randomization, concealment of treatment allocation, blinding, completeness of follow-up, and the use of intention-to-treat analysis.

\section{Statistical analysis}

We extracted the adjusted hazard ratio (HR) and corresponding 95\% confidence interval (CI) from each RCT to estimate the pooled $\mathrm{HR}$, and corresponding 95\% CI, for OS and PFS in the dual anti-HER2 therapy and monotherapy groups. We also allocated dichotomous frequency data to assess the pooled relative risks (RRs), and 
corresponding 95\% CI, of pCR and each AEs in the dual anti-HER2 therapy and monotherapy groups. Included in the analysis were five three-arm trials, with two antiHER2 monotherapy arms and one dual anti-HER2 therapy study. The two anti-HER2 monotherapy arms were merged into one group by adding the sample size and the number of events in each arm. Subgroup analyses were performed according to the addition of chemotherapy and the component drugs of dual anti-HER2 therapy (trastuzumab + lapatinib or trastuzumab + pertuzumab). When there was no statistically significant heterogeneity, a pooled effect was calculated using a fixed-effect model; otherwise, a random-effect model was employed. Heterogeneity of treatment effects between trials was evaluated using the chi-square $\left(\mathrm{X}^{2}\right)$ test and I-squared $\left(\mathrm{I}^{2}\right)$ statistic [18]. We also assessed the probability of publication bias using the Egger's [19] and Begg-Mazumdar [20] tests. Statistical analyses were carried out using STATA 11.0 (State Corporation, Lake Way, Texas, USA). A two-tailed $p$ value $<0.05$ was considered statistically significant.

\section{Results}

\section{Search results and trial characteristics}

We identified 737 potentially relevant trials from our initial electronic search, and excluded 685 trials after a preliminary review. The remaining 52 studies were retrieved for detailed assessment. Of these, 44 articles were excluded because they contained no combination therapy group, were phase II trials without a control arm, or they were not phase II or phase III studies. An additional article was included by searching ASCO 2012 Annual Meeting abstracts [21]. A further two studies were excluded because they were duplicates $[22,23]$; only the most recent reports were included [15,24]. Therefore, a total of seven [15,21,24-28] RCTs were included in our final analysis, which included data on 2,609 individual patients (Figure 1). The design characteristics of the included trials are presented in Table 1 . The role of dual anti-HER2 therapy in the neoadjuvant setting was evaluated in five trials [21,25-28] and two trials [15,24] investigated dual antiHER2 therapy in the metastatic setting. All the breast cancer patients were HER2-positive. The quality of the trials was assessed according to the pre-determined criteria of the Jadad score. Overall, three trials had a Jadad score of 5 $[23,26,27]$, three trials had a score of $4[21,25,28]$, and one trial had a score of 3 [24].

\section{PCR in neoadjuvant studies}

pCR was evaluated in a total of five trials [21,25-28], all which investigated the effect of anti-HER2 therapy in the neoadjuvant settings. In these trials, anti-HER2 agents were combined with chemotherapy: paclitaxel in three trials [21,26,27], FEC in one trial [28], and docetaxel in the remaining one trial [25]. There was also one arm without chemotherapy in the Neo-Sphere trial [25], and this arm was excluded in the pooled analysis. The pooled $\mathrm{pCR}$ rate

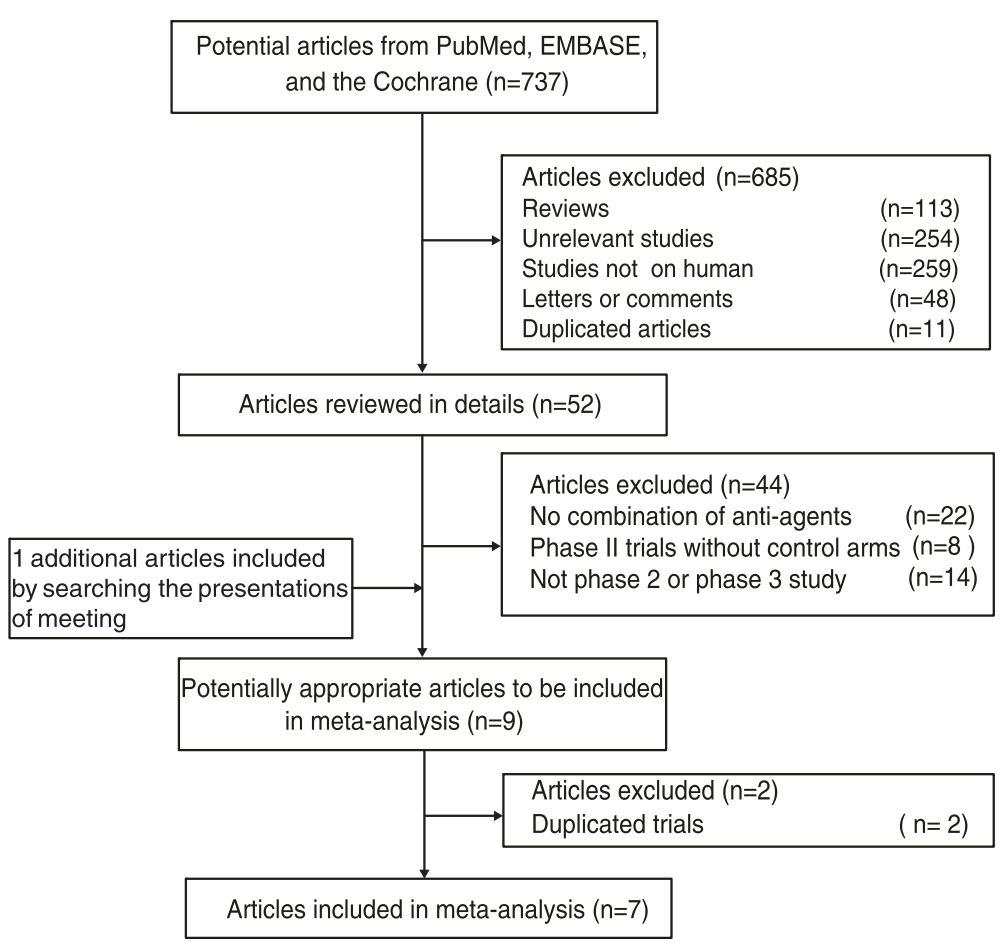

Figure 1 Flow diagram of the trial search and selection process. 
Table 1 Character of the included studies

\begin{tabular}{|c|c|c|c|c|c|c|c|c|c|c|}
\hline Study & Phase & $\begin{array}{l}\text { Treatment } \\
\text { staus }\end{array}$ & $\begin{array}{l}\text { Treatment } \\
\text { arms }\end{array}$ & Pts no. & Dosage & Chemotherapy & Treatment duration & $\begin{array}{l}\text { Median } \\
\text { age }\end{array}$ & $\begin{array}{l}\text { Jada } \\
\text { score }\end{array}$ & $\begin{array}{l}\text { Follow- } \\
\text { up }(\mathrm{m})\end{array}$ \\
\hline \multirow{3}{*}{$\begin{array}{l}\text { CHER-LOB } 26 \\
(2012)\end{array}$} & 2 & Neoadjuvant & $T$ & 36 & 2 mg/kg weekly (loading 4 mg/kg) & Paclitaxel & 26 weeks & 50 & 5 & NA \\
\hline & & & L & 39 & 1500 mg daily & Paclitaxel & 26 weeks & 49 & & NA \\
\hline & & & $L+T$ & 46 & 1000 mg daily + 2 mg/kg weekly (loading 4 mg/kg) & Paclitaxel & 26 weeks & 49 & & NA \\
\hline \multirow{3}{*}{$\begin{array}{l}\text { NeoALTTO } \\
(2012)\end{array}$} & 3 & Neoadjuvant & L & 149 & 1500 mg daily & Paclitaxel & 18 weeks & 50 & 5 & NA \\
\hline & & & T & 154 & 2 mg/kg weekly (loading 4 mg/kg) & Paclitaxel & 18 weeks & 49 & & NA \\
\hline & & & $L+T$ & 152 & 1000 mg daily + 2 mg/kg weekly (loading 4 mg/kg) & Paclitaxel & 18 weeks & 50 & & NA \\
\hline \multirow{3}{*}{$\begin{array}{l}\text { NSABP B-41 } 21 \\
(2012)\end{array}$} & 3 & Neoadjuvant & T & 177 & 2 mg/kg weekly (loading 4 mg/kg) & Paclitaxel & 12 weeks & NA & 4 & NA \\
\hline & & & L & 171 & 1250 mg daily & Paclitaxel & 13 weeks & NA & & NA \\
\hline & & & $L+T$ & 171 & 750 mg daily + 2 mg/kg weekly (loading 4 mg/kg) & Paclitaxel & 14 weeks & NA & & NA \\
\hline \multirow{4}{*}{$\begin{array}{l}\text { NeoSphere }{ }^{25} \\
(2012)\end{array}$} & 2 & Neoadjuvant & T & 107 & 6 mg/kg q3w (loading 8 mg/kg) & Docetaxel & 12 weeks & 50 & 4 & NA \\
\hline & & & $T+P$ & 107 & $\begin{array}{l}6 \text { mg/kg q3w (loading } 8 \text { mg/kg) + } 420 \text { mg/kg q3w } \\
\text { (loading } 840 \text { mg/kg) }\end{array}$ & docetaxel & 13 weeks & 50 & & NA \\
\hline & & & $T+P$ & 107 & $\begin{array}{l}6 \text { mg/kg q3w (loading } 8 \text { mg/kg) +420 mg/kg q3w } \\
\text { (loading } 840 \text { mg/kg) }\end{array}$ & NO & 14 weeks & 49 & & NA \\
\hline & & & $P$ & 96 & 420 mg/kg q3w (loading 840 mg/kg) & docetaxel & 15 weeks & 49 & 4 & NA \\
\hline \multirow{3}{*}{$\begin{array}{l}\text { Holmes } \\
\text { (2012) }\end{array}$} & NA & Neoadjuvant & T & 33 & 2 mg/kg weekly (loading 4 mg/kg) & FEC & 26 weeks & NA & & NA \\
\hline & & & $L$ & 34 & 1250 mg daily & FEC & 27 weeks & NA & & NA \\
\hline & & & $L+T$ & 33 & 750 mg daily + 2 mg/kg weekly & FEC & 28 weeks & NA & & NA \\
\hline \multirow[t]{2}{*}{$\begin{array}{l}\text { EGF10490024 } \\
(2012)\end{array}$} & 3 & Metastatic & $L+T$ & 148 & 1000 mg daily + 2 mg/kg weekly & No & $\begin{array}{l}\text { Until progression or } \\
\text { unacceptable toxicity }\end{array}$ & 51 & 3 & 12.8 \\
\hline & & & L & 148 & 1500 mg daily & No & $\begin{array}{l}\text { Until progression or } \\
\text { unacceptable toxicity }\end{array}$ & 51 & & 8.7 \\
\hline \multirow[t]{2}{*}{$\begin{array}{l}\text { CLEOPATRA }{ }^{15} \\
(2012)\end{array}$} & 3 & Metastatic & $P+T$ & 402 & $\begin{array}{l}6 \text { mg/kg q3w (loading } 8 \text { mg/kg) + } 420 \text { mg/kg q3w } \\
\text { (loading } 840 \text { mg/kg) }\end{array}$ & Docetaxel & $\begin{array}{l}\text { Until progression or } \\
\text { unacceptable toxicity }\end{array}$ & 54 & 5 & 30 \\
\hline & & & Placebo + T & 406 & 6 mg/kg q3w (loading 8 mg/kg) & Docetaxel & $\begin{array}{l}\text { Until progression or } \\
\text { unacceptable toxicity }\end{array}$ & 54 & & 30 \\
\hline
\end{tabular}

Abbreviations: $T$ Trastuzumab, L Lapatinib, $P$ Pertuzumab, pts no patients number, $m g$ milligram, $k g$ kilogram, Pacl Paclitaxel, FEC Fluorouracil (5FU), epirubicin, and cyclophosphamide; $q 3 \mathrm{w}$ three-weekly,

NA Not available, $m$ month. 
was $54.8 \%$ (278 of 508 patients; 95\% CI, 0.46-0.63) in the dual therapy group compared with $35.6 \%$ (442 of 995 patients; 95\% CI, 0.24-0.50) in the monotherapy group. The difference in pCR between dual agents and single antiHER2 agents was significant $(R R, 1.56$; 95\% CI, 1.23-1.97; $\mathrm{p}<0.001$ ), with no evidence of significant publication bias (Egger's test, $\mathrm{P}=0.624$; Begg-Mazumdar test, $\mathrm{P}=0.9370$; Figure 2).We noted some evidence of heterogeneity in the magnitude of this effect across the included studies ( $\mathrm{p}=$ $0.006, \mathrm{I}^{2}=72.3 \%$, which was mostly attributable to inclusion of the NSABP B-41 study [21]. A sensitivity analysis that excluded the NSABP B-41 study resulted in a similar RR $(1.74 ; 95 \%$ CI, 1.49-2.03; $\mathrm{p}<0.001)$ with much reduced heterogeneity $\left(\mathrm{p}=0.67, \mathrm{I}^{2}=0.00 \%\right)$.

\section{PFS and OS in metastatic studies or setting}

In our analysis, two studies [15,24] investigated the effect of dual anti-HER2 therapy in the metastatic setting. The CLEOPATRA study was a phase III study that included 808 patients with HER2-positive metastatic breast cancer [15]. In this study, patients were randomized to first-line treatment of pertuzumab + trastuzumab + docetaxel (dual anti-HER2 therapy group) or trastuzumab + docetaxel + placebo (control group). The median PFS was 18.7 months in the dual anti-HER2 therapy group and 12.4 months in the control group. The difference in PFS was significant (HR, 0.69; 95\% CI, 0.58-0.81; P<0.001). Furthermore, a significant benefit in OS was also observed in patients allocated to the dual anti-HER2 therapy treatment group compared with individuals assigned to the control group (HR, 0.66; 95\% CI $0.52-0.84 ; \mathrm{p}<0.001$ ). The median OS for patients allocated to the control group was 37.6 months but was not reached in the dual anti-HER2 therapy group. Another phase III study (EGF104900) enrolled patients with HER2-positive metastatic breast cancer whose disease had progressed during prior trastuzumab therapy
[24]. In this study, 296 patients were randomly assigned to receive either lapatinib + trastuzumab (dual anti-HER2 therapy group) or lapatinib monotherapy (control group). The median PFS was 11.1 and 8.1 weeks in the dual antiHER2 therapy and control groups, respectively. Median OS was 14 months for the dual anti-HER2 therapy group and 9.5 months for the lapatinib alone group. The dual anti-HER2 therapy group showed significant improvements in PFS (HR, 0.74; 95\% CI, 0.58-0.94; P = 0.011) and OS (HR, 0.74; 95\% CI, 0.57-0.97; P = 0.026). Although these two trials had different patient populations and settings, both showed a significant improvement in OS and PFS with dual anti-HER2 therapy.

\section{Subgroup analysis}

Subgroup analyses were performed to determine whether the type of neoadjuvant chemotherapy influenced the efficacy of dual anti-HER2 therapy. We found that the benefit of dual anti-HER2 therapy on pCR was similar among different chemotherapies. Detailed information regarding these analyses is summarized in Table 2 .

We also performed subgroup analyses based on the component drugs of dual anti-HER2 therapy (trastuzumab + lapatinib or trastuzumab + pertuzumab). The results of this subgroup analysis indicated that there were improvements in pCR in dual anti-HER2 therapies consisting of trastuzumab + lapatinib (RR, 1.52; 95\% CI, 1.152.01) and trastuzumab + pertuzumab (RR, 1.72; 95\% CI, 1.27-2.34; Table 2). Compared with monotherapy, dual anti-HER2 therapy consisting of trastuzumab + lapatinib showed a decrease in the HR for disease progression and death of $26 \%$ and $29 \%$, respectively. Similar benefits in PFS (HR, 0.69; 95\% CI, 0.58-0.81) and OS (HR, 0.66; 95\% CI, 0.52-0.84) were also observed with trastuzumab + pertuzumab dual anti-HER2 therapy.

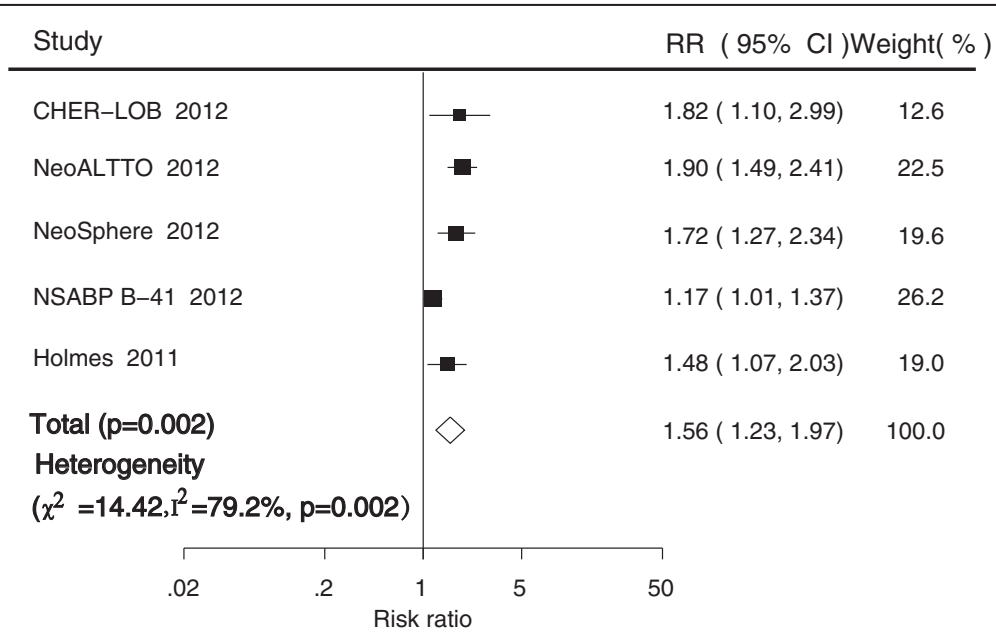

Figure 2 Meta-analysis of pathologic complete response between dual anti-HER2 therapy and monotherapy groups. 
Table 2 Subgroup analysis based on the type of chemotherapy and the component of dual agents

\begin{tabular}{lcc}
\hline Subgroup & Reference & PCR \\
\hline The type of Chemotherapy & $21,25,26,27,28$ & $1.56(1.23,1.97)$ \\
Paclitaxel & $21,26,27$ & $1.55(1.06,2.28)$ \\
FEC & 28 & $1.48(1.07,2.03)$ \\
Docetaxel & 25 & $1.72(1.27,2.34)$ \\
Component of dual agents & & \\
L+T & $21,26,27,28$ & $1.52(1.15,2.01)$ \\
$P+T$ & 25 & $1.72(1.27,2.34)$ \\
\hline
\end{tabular}

Abbreviations: $T$ Trastuzumab, $L$ Lapatinib, $P$ Pertuzumab.

\section{Relative risk of AEs}

The use of dual anti-HER2 therapy was associated with an increase in serious AEs (RR, 1.22; 95\% CI, 1.03-1.46; $\mathrm{p}=0.024)$. In addition, dual anti-HER2 therapy increased the risk of grade 3-4 diarrhea (RR, 1.64; 95\% CI, 1.262.13; $\mathrm{p}<0.001$ ), all grades of dermatologic toxicity (RR, 1.41; 95\% CI, 1.19-1.68; $\mathrm{p}<0.001$ ), and febrile neutropenia (RR, 1.55; 95\% CI, 1.08-2.23; $\mathrm{p}=0.143$ ). No statistically significant differences were observed between the two arms for neutropenia (RR, 0.99; 95\% CI, 0.88-1.10; $\mathrm{p}=0.645)$, hepatic toxicity (RR, 1.01; 95\% CI, 0.66-1.55; $\mathrm{p}=0.818$ ), grade $3-4$ dermatologic toxicity ( $R R, 1.28$; 95\% CI, 0.73-2.23; $\mathrm{p}=0.378)$, nausea $(\mathrm{RR}, 1.04 ; 95 \% \mathrm{CI}$, $0.90-1.20 ; \mathrm{p}=0.597)$, or fatigue (RR, 1.01; $95 \% \mathrm{CI}, 0.86-$ $1.19 ; \mathrm{p}=0.879$; Figure 3 ).

\section{Cardiac toxicity}

The risk of heart failure was reported in six trials [15,21,24-27]. Overall, there was no statistically significant difference in the risk of heart failure between dual anti-HER2 therapy and monotherapy (RR, 0.79; 95\% CI, $0.23-2.68 ; \mathrm{p}=0.708)$. There were five trials $[15,24-27]$ that reported data for left ventricular ejection fraction (LVEF) decline, but no statistically significant difference in risk between the treatment groups was found ( $R R$, 1.12; 95\% CI, 0.51-2.44; $\mathrm{p}=0.773$; Figure 4).

\section{Discussion}

This systematic review summarizes all the available published, randomized evidence on the efficacy and safety of dual anti-HER2 therapy compared with monotherapy in HER2-positve breast cancer patients. Preclinical studies have suggested that combining different HER2 inhibitors with complementary mechanisms of action can help maximize the suppression of oncogenic processes involved in disease progression. Consequently, an increasing number of clinical trials have been performed to investigate this promising therapeutic approach $[15,24,25]$. Combining data from all the currently published trials, this systematic review confirms the benefit of dual HER2directed therapy over monotherapy for HER2-positve breast cancer.

By pooling the data from the included trials, we showed that dual anti-HER2 therapy significantly improves pCR

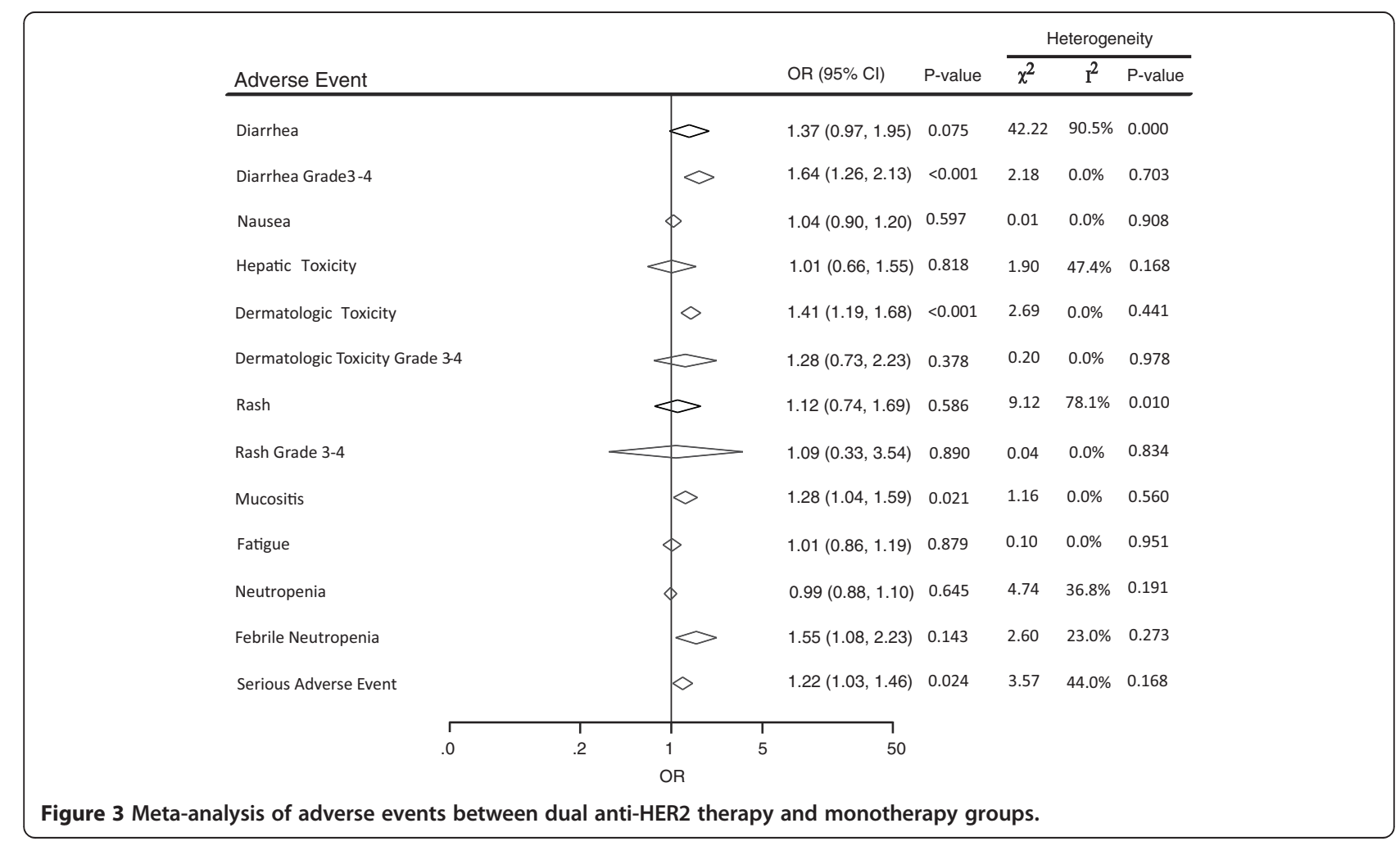




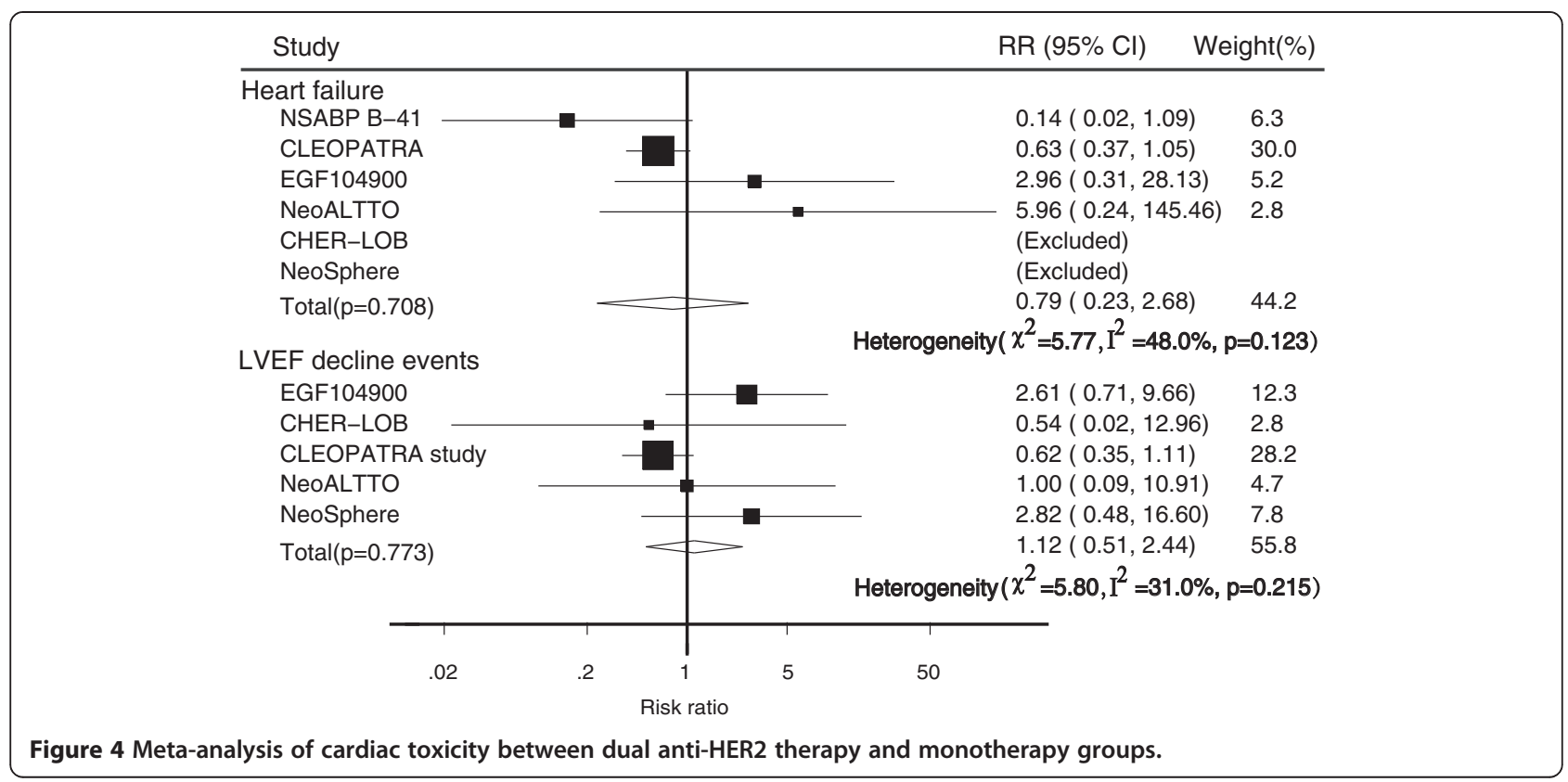

rate in the neoadjuvant setting (RR, 1.56; 95\% CI, 1.231.97; $\mathrm{p}<0.001)$. Hence, dual anti-HER2 therapy might be an attractive strategy in this setting. In the metastatic setting, dual anti-HER2 therapy, compared to monotherapy, demonstrated significant benefits for OS and PFS in the EGF104900 and CLEOPATRA studies. However, because of the limited data regarding metastatic disease, these benefits require further study.

Despite the advantages of dual anti-HER2 therapy in the neoadjuvant settings, a number of questions have arisen: 1) does the type of chemotherapy alter the efficacy of dual anti-HER2 therapy; and 2) which combination of the HER2 inhibitors has optimal efficacy for HER2-positive breast cancer. In this study, subgroup analyses were conducted to address these questions. When grouped by the type of chemotherapy, the improvement in the $\mathrm{pCR}$ rate with dual anti-HER2 therapy was similar, regardless of the specific chemotherapies used. Furthermore, similar efficacy was observed with trastuzumab + pertuzumab and trastuzumab + lapatinib. Based on current data, it appears that the type of chemotherapy and specific components of dual antiHER2 therapy have little impact on the efficacy of the treatment. However, due to the limited number of trials in each subgroup, these results need to be validated in further studies.

Our study also raises some safety concerns. As the HER2 signaling pathway plays an important role in cardiac physiology [29], we investigated the risk of cardiac AEs. We found that no increased risk of cardiac toxicity was associated with dual anti-HER2 therapy, which is consistent with the results of a previous study conducted by Valachis et al. [30]. However, we found that some other toxicities were more frequent in patients who received dual anti-HER 2 therapy, particularly grade 3-4 diarrhea, dermatologic toxicity, mucosis, febrile neutropenia, and other serious AEs. Clinicians should take note of this, since the incidence of AEs substantially affects a patient's quality of life, and may lead to discontinuation of dual treatment.

Several limitations of this systematic review should be acknowledged. Firstly, data from the ongoing ALTTO and MARIANNE [14] trials are still unavailable. Secondly, our results are based on published data and presented clinical trials; individual patient data were not unavailable. Thirdly, because the results of this metaanalysis are confined to trastuzumab + lapatinib or trastuzumab + pertuzumab, the results are not necessarily applicable to treatments using other drug combinations. In addition, some subgroup analyses were based on limited studies, and the results should be interpreted with caution.

\section{Conclusions}

This systematic review shows that, according to the present available data, dual anti-HER2 therapy seems to be more effective than monotherapy in the neoadjuvant setting. In the metastatic setting, limited data is available and further evaluation of the role of dual anti-HER2 treatment is required. However, it is reasonable to believe that a shift towards a dual therapeutic approach will yield clinically meaningful improvements for patients with HER2-postive breast cancer. However, given the increased risk of AEs associated with dual anti-HER2 therapy, it is important for health care practitioners to be aware of the potential risks and to provide close monitoring to improve patient outcome. 


\section{Competing interests}

The authors declare that they have no competing interests.

\section{Authors' contributions}

Conception/design: JH, X-JZ, XZ. Collection and/or assembly of data: XZ, F-FY. Data analysis and interpretation: XZ, XW, Y-SL. Manuscript writing: XZ, $X-J Z, T-Y Z$. All authors read and approved the final manuscript.

\section{Acknowledgements}

This work is supported by the grants from the National Natural Science Foundation of China (NO. 81373105), the Key Discipline Construction of Evidence-based Public Health in Shanghai (NO. 12GWZX0602), The Ministry of Science and Technology of China (Project NOs. 2009ZX09312-025, 2008ZX09312-007, and 2011ZXJ09202-015), and the Fund for Youth in Second Military Medical University (NO. 2012QN10). The funders had no role in the study design, data collection, analysis, decision to publish, or preparation of the manuscript. The corresponding author has full access to all the data in the study and had final responsibility for the decision to submit for publication.

\section{Author details}

${ }^{1}$ Department of Health Statistics, Second Military Medical University, Shanghai, China. ${ }^{2}$ Renji Hospital, Shanghai Jiao Tong University School of Medicine, Shanghai, China. ${ }^{3}$ Department of Special Treatment, Eastern Hepatobiliary Hospital, Second Military Medical University, Shanghai, China.

Received: 12 March 2014 Accepted: 21 August 2014

Published: 28 August 2014

\section{References}

1. Brollo J, Curigliano G, Disalvatore D, Marrone BF, Criscitiello C, Bagnardi V, Kneubil MC, Fumagalli L, Locatelli M, Manunta S, Goldhirsch A: Adjuvant trastuzumab in elderly with HER-2 positive breast cancer: a systematic review of randomized controlled trials. Cancer Treat Rev 2013, 39(1):44-50.

2. Patani N, Mokbel K: Herceptin and breast cancer: an overview for surgeons. Surg Oncol 2010, 19(1):e11-e21.

3. Binns C, Low WY, Lee MK: Breast cancer: an increasing public health problem in the Asia pacific region. Asia Pac J Public Health 2013, 25(5):364-367.

4. Ross JS, Slodkowska EA, Symmans WF, Pusztai L, Ravdin PM, Hortobagyi GN: The HER-2 receptor and breast cancer: ten years of targeted anti-HER-2 therapy and personalized medicine. Oncologist 2009, 14(4):320-368.

5. Vogel UF: Confirmation of a low HER2 positivity rate of breast carcinomas - limitations of immunohistochemistry and in situ hybridization. Diagn Pathol 2010, 5:50.

6. Burris HA 3rd, Rugo HS, Vukelja SJ, Vogel CL, Borson RA, Limentani S, Tan-Chiu E, Krop IE, Michaelson RA, Girish S, Amler L, Zheng M, Chu YW, Klencke B, O'Shaughnessy JA: Phase II study of the antibody drug conjugate trastuzumab-DM1 for the treatment of human epidermal growth factor receptor 2 (HER2)-positive breast cancer after prior HER2-directed therapy. J Clin Oncol 2011, 29(4):398-405.

7. Tonyali O, Benekli M, Berk V, Coskun U, Ozkan M, Yildiz R, Ucgul E, Sevinc A, Uncu D, Demirci U, Buyukberber S: Efficacy and toxicity of Trastuzumab and Paclitaxel plus Capecitabine in the first-line treatment of HER2positive metastatic breast cancer. J Cancer Res Clin Oncol 2013, 139(6):981-986.

8. Rimawi MF, Mayer IA, Forero A, Nanda R, Goetz MP, Rodriguez AA, Pavlick AC, Wang T, Hilsenbeck SG, Gutierrez C, Schiff R, Osborne CK, Chang JC: Multicenter phase II study of neoadjuvant lapatinib and trastuzumab with hormonal therapy and without chemotherapy in patients with human epidermal growth factor receptor 2-overexpressing breast cancer: TBCRC 006. J Clin Oncol 2013, 31(14):1726-1731.

9. Geyer CE, Forster J, Lindquist D, Chan S, Romieu CG, Pienkowski T, JagielloGruszfeld A, Crown J, Chan A, Kaufman B, Skarlos D, Campone M, Davidson N, Berger M, Oliva C, Rubin SD, Stein S, Cameron D: Lapatinib plus capecitabine for HER2-positive advanced breast cancer. N Engl J Med 2006, 355(26):2733-2743.

10. Xu BH, Jiang ZF, Chua D, Shao ZM, Luo RC, Wang XJ, Liu DG, Yeo W, Yu SY, Newstat B, Preston A, Martin AM, Chi HD, Wang L: Lapatinib plus capecitabine in treating HER2-positive advanced breast cancer: efficacy, safety, and biomarker results from Chinese patients. Chin J Cancer 2011, 30(5):327-335.

11. Scaltriti M, Eichhorn PJ, Cortes J, Prudkin L, Aura C, Jimenez J, Chandarlapaty S, Serra V, Prat A, Ibrahim YH, Guzman M, Gili M, Rodriguez O, Rodrlguez S, Perez J, Green SR, Mai S, Rosen N, Hudis C, Baselga J: Cyclin E amplification/ overexpression is a mechanism of trastuzumab resistance in HER2+ breast cancer patients. Proc Natl Acad Sci U S A 2011, 108(9):3761-3766.

12. Nagata $Y$, Lan KH, Zhou X, Tan M, Esteva FJ, Sahin AA, Klos KS, Li P, Monia BP, Nguyen NT, Hortobagyi GN, Hung MC, Yu D: PTEN activation contributes to tumor inhibition by trastuzumab, and loss of PTEN predicts trastuzumab resistance in patients. Cancer Cell 2004, 6(2):117-127.

13. Alvarez RH, Hortobagyi GN: Dual human epidermal growth factor receptor 2 blockade for the treatment of HER2-positive breast cancer. Breast Cancer 2013, 20(2):103-110.

14. Konecny GE: Emerging strategies for the dual inhibition of HER2-positive breast cancer. Curr Opin Obstet Gynecol 2013, 25(1):55-65.

15. Swain SM, Kim SB, Cortes J, Ro J, Semiglazov V, Campone M, Ciruelos E, Ferrero JM, Schneeweiss A, Knott A, Clark E, Ross G, Benyunes MC, Baselga J: Pertuzumab, trastuzumab, and docetaxel for HER2-positive metastatic breast cancer (CLEOPATRA study): overall survival results from a randomised, double-blind, placebo-controlled, phase 3 study. Lancet Oncol 2013, 14(6):461-471.

16. Moher D, Cook DJ, Eastwood S, Olkin I, Rennie D, Stroup DF: Improving the quality of reports of meta-analyses of randomised controlled trials: the QUOROM statement. Quality of reporting of meta-analyses. Lancet 1999, 354(9193):1896-1900.

17. Jadad AR, Moore RA, Carroll D, Jenkinson C, Reynolds DJ, Gavaghan DJ, McQuay $\mathrm{HJ}$ : Assessing the quality of reports of randomized clinical trials: is blinding necessary? Control Clin Trials 1996, 17(1):1-12.

18. Higgins JP, Thompson SG, Deeks JJ, Altman DG: Measuring inconsistency in meta-analyses. BMJ 2003, 327(7414):557-560.

19. Egger M, Davey Smith G, Schneider M, Minder C: Bias in meta-analysis detected by a simple, graphical test. BMJ 1997, 315(7109):629-634.

20. Begg CB, Mazumdar M: Operating characteristics of a rank correlation test for publication bias. Biometrics 1994, 50(4):1088-1101.

21. Robidoux A, Tang G, Rastogi P, Geyer CE, Azar CA, Atkins JN, Fehrenbacher L, Bear HD, Baez-Diaz L, Sarwar S, Margolese RG, Farrar WB, Brufsky AM, Shibata HR, Bandos H, Paik S, Costantino JP, Swain SM, Mamounas EP, Wolmark N: Lapatinib as a component of neoadjuvant therapy for HER2positive operable breast cancer (NSABP protocol B-41): an open-label, randomised phase 3 trial. J Clin Oncol 2013, 14(12):1183-1192.

22. Blackwell KL, Burstein HJ, Storniolo AM, Rugo H, Sledge G, Koehler M, Ellis C, Casey M, Vukelja S, Bischoff J, Baselga J, O'Shaughnessy J: Randomized study of Lapatinib alone or in combination with trastuzumab in women with ErbB2-positive, trastuzumab-refractory metastatic breast cancer. J Clin Oncol 2010, 28(7):1124-1130.

23. Baselga J, Cortes J, Kim SB, Im SA, Hegg R, Im YH, Roman L, Pedrini JL, Pienkowski T, Knott A, Clark E, Benyunes MC, Ross G, Swain SM: Pertuzumab plus trastuzumab plus docetaxel for metastatic breast cancer. N Engl J Med 2012, 366(2):109-119.

24. Blackwell KL, Burstein HJ, Storniolo AM, Rugo HS, Sledge G, Aktan G, Ellis C, Florance A, Vukelja S, Bischoff J, Baselga J, O'Shaughnessy J: Overall survival benefit with lapatinib in combination with trastuzumab for patients with human epidermal growth factor receptor 2-positive metastatic breast cancer: final results from the EGF104900 Study. J Clin Oncol 2012, 30(21):2585-2592.

25. Gianni L, Pienkowski T, Im YH, Roman L, Tseng LM, Liu MC, Lluch A, Staroslawska E, de la Haba-Rodriguez J, Im SA, Pedrini JL, Poirier B, Morandi P, Semiglazov V, Srimuninnimit V, Bianchi G, Szado T, Ratnayake J, Ross G, Valagussa P: Efficacy and safety of neoadjuvant pertuzumab and trastuzumab in women with locally advanced, inflammatory, or early HER2-positive breast cancer (NeoSphere): a randomised multicentre, open-label, phase 2 trial. Lancet Oncol 2012, 13(1):25-32.

26. Guarneri V, Frassoldati A, Bottini A, Cagossi K, Bisagni G, Sarti S, Ravaioli A, Cavanna L, Giardina G, Musolino A, Untch M, Orlando L, Artioli F, Boni C, Generali DG, Serra P, Bagnalasta M, Marini L, Piacentini F, D'Amico R, Conte $\mathrm{P}$ : Preoperative chemotherapy plus trastuzumab, lapatinib, or both in human epidermal growth factor receptor 2-positive operable breast cancer: results of the randomized phase II CHER-LOB study. J Clin Oncol 2012, 30(16):1989-1995. 
27. Baselga J, Bradbury I, Eidtmann H, Di Cosimo S, de Azambuja E, Aura C, Gomez H, Dinh P, Fauria K, Van Dooren V, Aktan G, Goldhirsch A, Chang TW, Horvath Z, Coccia-Portugal M, Domont J, Tseng LM, Kunz G, Sohn JH, Semiglazov V, Lerzo G, Palacova M, Probachai V, Pusztai L, Untch M, Gelber RD, Piccart-Gebhart M: Lapatinib with trastuzumab for HER2-positive early breast cancer (NeoALTTO): a randomised, open-label, multicentre, phase 3 trial. Lancet 2012, 379(9816):633-640.

28. Holmes FA, Nagarwala YM, Espina VA, Liotta LA, Danso MA, Gallagher RI, McIntyre K, Osborne CRC, Mahoney JM, Florance AM, Anderson TC, O'Shaughnessy J, Anderson TC, O'Shaughnessy J: Correlation of molecular effects and pathologic complete response to preoperative lapatinib and trastuzumab, separately and combined prior to neoadjuvant breast cancer chemotherapy. J Clin Oncol 2011, 29(suppl):abstr 506.

29. Zhao YY, Sawyer DR, Baliga RR, Opel DJ, Han X, Marchionni MA, Kelly RA: Neuregulins promote survival and growth of cardiac myocytes. Persistence of ErbB2 and ErbB4 expression in neonatal and adult ventricular myocytes. J Biol Chem 1998, 273(17):10261-10269.

30. Valachis A, Nearchou A, Polyzos NP, Lind P: Cardiac toxicity in breast cancer patients treated with dual HER2 blockade. Int J Cancer 2013, 133(9):2245-2252.

doi:10.1186/1471-2407-14-625

Cite this article as: Zhang et al:: Effect and safety of dual anti-human epidermal growth factor receptor 2 therapy compared to monotherapy in patients with human epidermal growth factor receptor 2-positive breast cancer: a systematic review. BMC Cancer 2014 14:625.

\section{Submit your next manuscript to BioMed Central} and take full advantage of:

- Convenient online submission

- Thorough peer review

- No space constraints or color figure charges

- Immediate publication on acceptance

- Inclusion in PubMed, CAS, Scopus and Google Scholar

- Research which is freely available for redistribution 